\title{
Sociobiology
}

RESEARCH ARTICLE - ANTS

\section{A Checklist of Ants (Hymenoptera: Formicidae) in Pakistan}

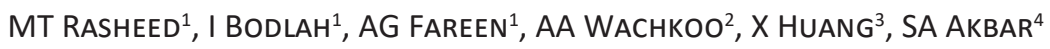 \\ 1 - Insect Biodiversity and Conservation group, Department of Entomology, Pir Mehr Ali Shah Arid Agriculture University, Rawalpindi, Pakistan \\ 2 - Department of Zoology, Government Degree College, Shopian, Jammu \& Kashmir, India \\ 3 - State key Laboratory of Ecological Pest Control for Fujian and Taiwan Crops, College of Plant Protection, Fujian Agriculture and Forestry \\ University, Fuzhou, China \\ 4 - Central Institute of Temperate Horticulture, Srinagar, India
}

\section{Article History \\ Edited by \\ Evandro N. Silva, UEFS, Brazil \\ Received 15 December 2018 \\ Initial acceptance 01 May 2019 \\ Final acceptance $\quad 04$ August 2019 \\ Publication date $\quad 14$ November 2019}

\section{Keywords}

Formicidae, Ants, Pakistan, Endemism, Tramp species, new records.

\section{Corresponding author Imran Bodlah \\ Insect Biodiversity and Conservation Group Department of Entomology \\ Pir Mehr Ali Shah Arid Agriculture University Rawalpindi, Pakistan \\ E-Mail: imranbodlah@gmail.com}

\begin{abstract}
The present paper provides an updated checklist of the ants (Hymenoptera: Formicidae) of Pakistan. These include seven of the 21 known extant subfamilies with 103 valid ant species in 35 genera. Five species are reported for the first time from Pakistan: Cardiocondyla wroughtonii Forel, 1890; Crematogaster biroi Mayr, 1897; Ooceraea biroi (Forel, 1907); Pseudoneoponera rufipes Jerdon, 1851 and Strumigenys godeffroyi Mayr, 1866. Images to scientifically validate new faunal records from Pakistan and facilitate prompt identification are provided. Among the newly recorded species, three species viz., C. wroughtonii; $O$. biroi and $S$. godeffroyi are considered as tramp species having the cosmopolitan distribution. Notes about type localities, depositories and distribution in Pakistan are provided to each species record. The list provides a synthesis of the regional taxonomical work carried out until now and will serve as a baseline survey for future studies.
\end{abstract}

\section{Introduction}

Pakistan occupies 882,000 sq. km, lying between 24 and 37 degrees north and 61 and 75 degrees east. The country straddles three of the world's eight biogeographic realms: IndoMalayan, Palearctic, and AfricoTropical and four of Earth's ten biomes: desert, temperate grassland, tropical seasonal forest and mountain (Cox \& Moore, 1993). Almost $80 \%$ of the total land area of the country is represented by mountanious arid and semi-arid regions, which support abrupt changes in altitude generating many changes in species composition within short distances (Anwar \& Shank, 2002). The country has rich biodiversity and is signatory to virtually all the important international biodiversity agreements (GOP, 1999). Still, estimates on biodiversity are far from conclusive. In addition to this, serious issues like deforestation, overgrazing, soil erosion, waterlogging, continuing loss of forest habitat, are posing major threats to the biodiversity of the country (Baig \& Al-Subaiee, 2011). An efficient workable strategy should be to conserve, attain sustainable levels of biodiversity and prioritise keystone species groups for drafting of conservational strategies.

Ants are one of the major keystone species, representing about $30 \%$ of terrestrial faunal biomass globally, and evolved as one of the most species-rich and ecologically diverse group of social insects (Andersen \& Majer, 2004). Ants perform numerous functions as predators, prey, detritivores, mutualists, herbivores, aid in nutrient mineralization; its movement and distribution, communition, soil aggregate formation, seed dispersal and tunneling (Folgarait, 1998; Pfeiffer et al., 2013) and as such are essential for proper functioning of most terrestrial ecosystems and resulting ecosystem services (Del Toro et al., 2012). Ants are more sensitive than other insects to ecosystem change and are often 
used in environmental monitoring studies to assess ecosystem change on biodiversity (Paknia \& Pfeiffer, 2011). Ants are also useful in natural areas restoration efforts because of their fast response to changes in habitat quality (Andersen et al., 2006; Gibb et al. 2015).

Little information is available on the ants of Pakistan with a most comprehensive account been a 115 years old compilation of Bingham (1903). The study stands outdated in modern context due to several taxonomic regroupings, unrecognized synonymies and other zoogeographical confusions. Compilation of Menozzi, (1939), of the Italian expedition led by S.A.R. il Duca di Spoleto in Himalayan and Karakorum is much pertinent to the present study. The study was carried in the entire Himalayan belt of which, now parts are governed by India, Pakistan and China separately. Most of the Pakistan ant records are from Gilgit-Baltistan region during this expedition. Other papers pertinent include Forel (1904); Donisthorpe (1933); Wilson (1955); Brown (1959);
Eidmann (1942); Mani and Singh (1962); Dlussky (1965); Pisarski (1967); Collingwood (1982). Recent contributions focused on systematic accounts and species enumeration include those of Seifert (1992, 2003, 2008); Radchenko and Elmes (1999, 2001, 2010); Ward (2001); Aslam et al. (2006); Li-zhong (2006); Csösz et al. (2007); Seifert and Schultz (2009); Yamane (2009); Umair et al. (2012); Wetterer (2009, 2012); Laciny et al. (2015); Bharti et al (2016); Bodlah et al. (2016); Janicki et al. (2016); Bodlah et al. (2017a); Bodlah et al. (2017b); Seifert et al. (2017); Guénard et al. (2017).

Recognizing the importance of ants as major ecosystem players and scatter of the available literature, we herewith provide a first ever comprehensive account of the regional ants of Pakistan. The paper summarises all the old and new information available on the group. The paper provides baseline data against which futuristic faunal changes could be assessed with respect to perturbations and will also help to frame future conservation programs.

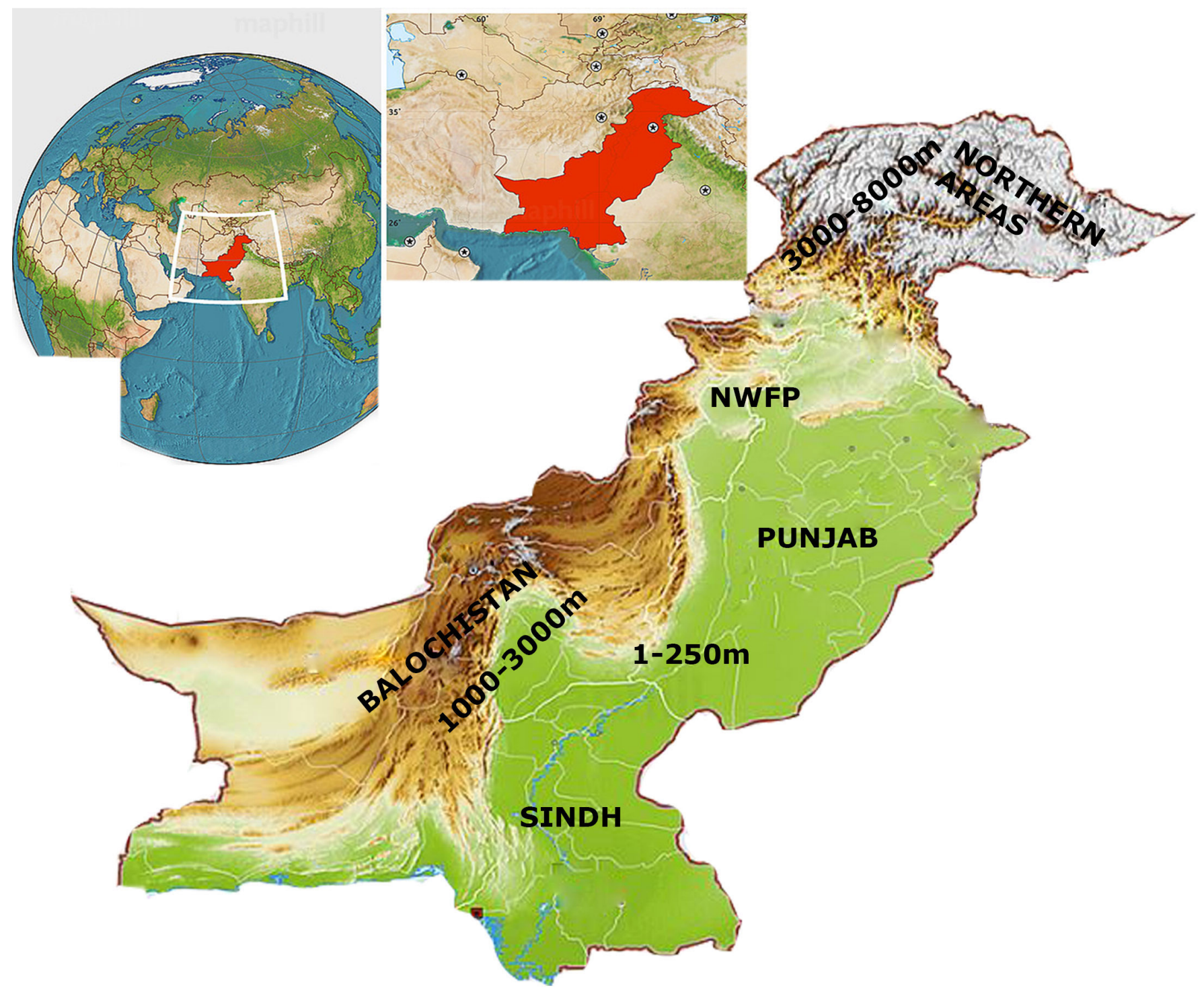

Fig 1. Map of Pakistan. 


\section{Material and Methods}

The checklist is based on available literature, museum collections and recent ant collection across various localities of Pakistan. New ant records from Pakistan were studied under Labomed CZM6 microscope. Images were prepared with a digital camera (Amscope 18 megapixels, model number MU1803) attached with same microscope using Helicon Focus stacking software and later cleaned in Adobe Photoshop CS6 software. Specimens were identified upto genus and species levels, using most recent relevant literature. Names of described species presented are in accordance with the most recent classification following AntWeb (2019). Genera and species names are arranged in alphabetical order following each subfamily, to make easier the search for a given taxon. References of original descriptions and local distribution are listed for all species. The acronyms used for collections are listed below:

AMNH - American Museum of Natural History, New York

ANIC - Australian National Insect Collection, Canberra, Australia BMNH - Natural History Museum, London, United Kingdom FMNH - Field Museum of Natural History, Chicago

HNHM - Hungarian Natural History Museum, Budapest, Hungary MHNG - Muséum d'Histoire Naturelle, Geneva, Switzerland MNHN - Muséum National d'Histoire Naturelle, Paris, France MSNG - Museo Civico di Storia Naturale "Giacomo Doria", Genova, Italy

MSNM - Museo Civico di Storia Naturale, Milano, Italy

MZPW - Museum of the Zoological Institute of the Polish Academy of Sciences Warsaw, Poland

NHMB - Naturhistorisches Museum, Basel, Switzerland

NHMW - Naturhistorisches Museum, Wien, Austria

NMNL - National Museum of Natural History Naturalis, Leiden OUMNH - University Museum of Natural History, Oxford, United Kingdom

PUAC - Punjabi University Patiala Ant Collection, Punjab, India SMNG - Senckenberg Museum für Naturkunde Görlitz, Görlitz UNK - Unknown depository of type material

ZISP - Zoological Institute, Academy of Sciences, St. Petersburg, Russia

ZMHB - Museum für Naturkunde der Humboldt-Universität, Berlin, Germany

\section{Results}

\section{DOLICHODERINAE}

Chronoxenus myops (Forel, 1895)

Bothriomyrmex myops Forel, 1895: 471. TL: South Konkan, Maharashtra: India [Lectotype: MHNG].

Distribution: Kashmir (Menozzi, 1939: 338).

Tapinoma melanocephalum (Fabricius, 1793)

Formica melanocephala Fabricius, 1793: 353. TL: Cayenne, French Guiana [Type: UNK].

Distribution: Karachi (Wetterer, 2009: 25).

\section{Tapinoma wroughtonii Forel, 1904}

Tapinoma wroughtonii Forel, 1904: 26. TL: Jhelum valley, Jammu and Kashmir: India [Syntype: MHNG].

Distribution: Jhelum valley, Kashmir (Forel, 1904: 26; Menozzi, 1939: 338); Gor, Gilgit-Baltistan (Eidmann, 1942: 249).

\section{DORYLINAE}

\section{Aenictus sagei Forel, 1901}

Aenictus wroughtonii var. sagei Forel, 1901a: 469. TL: Dharamsala, Himachal Pradesh: India [Lectotype: MHNG].

Distribution: Torkham, Peshawar (Pisarski, 1967: 377).

Dorylus fulvus (Westwood, 1839)

Typhlopone fulva Westwood, 1839: 219. TL: North Africa [Holotype: OUMNH].

Distribution: Peshawar (FMNHINS0000045249: AntWeb, 2019).

\section{Dorylus labiatus Shuckard, 1840}

Dorylus labiatus Shuckard, 1840: 319. TL: India [Syntype: OUMNH].

Distribution: Islamabad, Rawalpindi (Aslam et al., 2006: 86).

\section{Lioponera longitarsus Mayr, 1879}

Lioponera longitarsus Mayr, 1879: 667. TL: [Ostind.]: India [Syntype: NHMW].

Distribution: Islamabad, Rawalpindi (Umair et al., 2012: 703).

\section{Ooceraea biroi (Forel, 1907) Figures (2-4)}

Cerapachys (Syscia) biroi Forel, 1907: 7. TL: Singapore [Lectotype: MHNG].
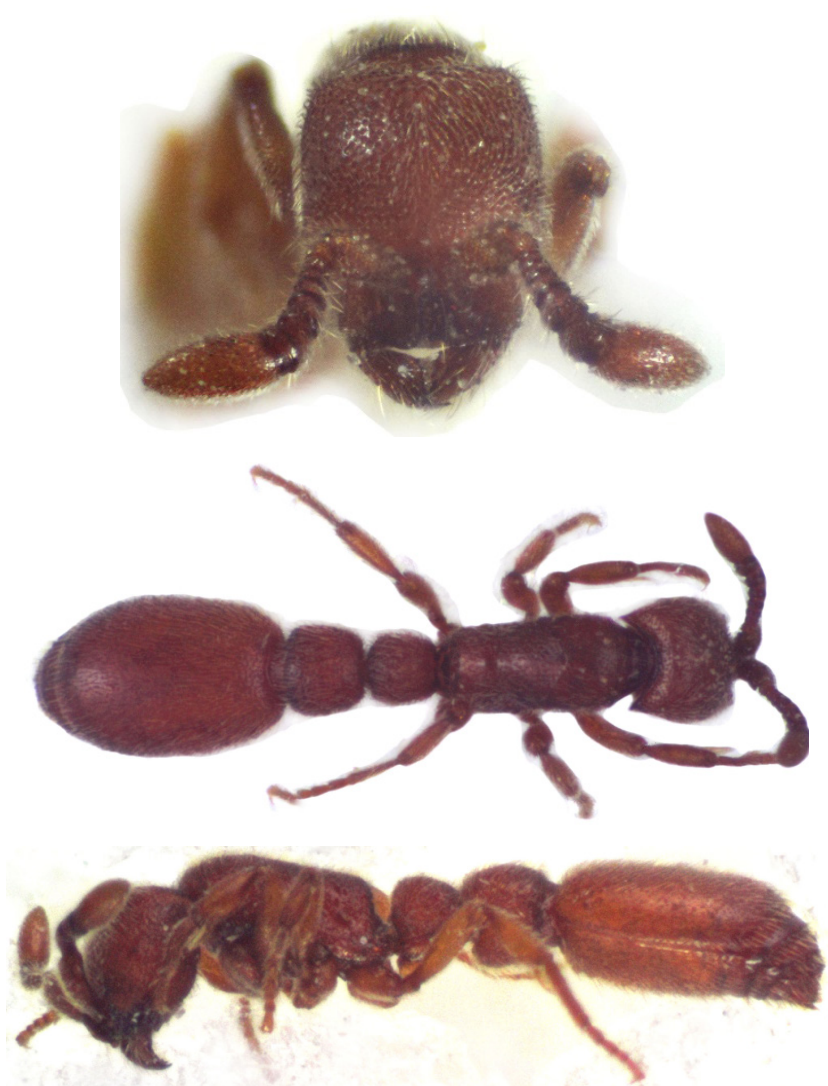

Figures 2-4. Ooceraea biroi (Forel, 1907): (2) Head, frontal view; Habitus (3) dorsal view; (4) lateral view. 
Material examined. Pakistan, Islamabad, Kachnar Park Forest area, 3340.602'N 7304.574'E, 603 m.a.s.1., 65 (workers), 20.xi.2015, 25 (workers), 24.viii.2016, 25 (workers), 12.ix.2017, under stone, leg. M.T. Rasheed.

Distribution: Pakistan (new record).

\section{FORMICINAE}

\section{Camponotus buddhae Forel, 1892}

Camponotus buddhae Forel, 1892a: 238. TL: Lahoul front Thibet, Himachal Pradesh: India [Syntype: MHNG].

Distribution: Askole, Gilgit-Baltistan (Menozzi, 1939: 315);

Nanga Parbat, Gilgit-Baltistan (Eidmann, 1942: 251).

Camponotus compressus (Fabricius, 1787)

Formica compressa Fabricius, 1787: 307. TL: Tharangambadi, Tamil Nadu: India [Type: UNK].

Distribution: Islamabad, Rawalpindi (Umair et al., 2012: 703).

Camponotus confucii Forel, 1894

Camponotus confucii Forel, 1894: 396. TL: Kanara, Karnataka: India [Syntypes: MHNG].

Distribution: Rawalpindi (Umair et al., 2012: 702).

\section{Camponotus japonicus Mayr, 1866}

Camponotus japonicus Mayr, 1866a: 885. TL: Japan [Syntype: NMNL].

Distribution: Turbaling, Gilgit-Baltistan (Eidmann, 1942: 251; Yasumatsu \& Brown, 1957: 47); Islamabad, Rawalpindi (Umair et al., 2012: 703).

\section{Camponotus oblongus (Smith, 1858)}

Formica oblonga Smith, 1858: 21. TL: [Birmah] Myanmar; [Holotype: BMNH].

Distribution: Rawalpindi (Umair et al., 2012: 702).

Camponotus sericeus (Fabricius, 1798)

Formica sericea Fabricius, 1798: 279. TL: Senegal [Type: UNK]. Distribution: Islamabad, Rawalpindi (Umair et al., 2012: 702).

Camponotus socrates Forel, 1904

Camponotus socrates Forel, 1904: 27. TL: Jhelum valley, Jammu and Kashmir: India [Syntypes: MHNG].

Distribution: Kashmir (Forel, 1904: 29).

Camponotus sylvaticus basalis Smith, 1878

Camponotus basalis Smith, 1878: 9. TL: Sindh valley, Jammu and Kashmir: India [Holotype: UNK].

Distribution: Tolti Kharmang, Gilgit-Baltistan (Menozzi, 1939: 315); Turbaling, Gilgit-Baltistan (Eidmann, 1942: 251).

\section{Camponotus sylvaticus paradichrous Emery, 1925}

Camponotus sylvaticus var. paradichroa Emery, 1925: 101. TL: Himalaya: India [Type: UNK].

Distribution: Askole, Braldu, Gilgit-Baltistan (Menozzi, 1939: 315; Mani \& Singh, 1962: 84).
Camponotus variegatus dulcis Dalla Torre, 1893

Camponotus mitis var. dulcis Dalla Torre, 1893: 243. TL: Bhamò [Birmania]: Myanmar [Syntype: MSNG].

Distribution: Razmak, Waziristan (CASENT0906973: AntWeb, 2019).

Cataglyphis aenescens (Nylander, 1849)

Formica aenescens Nylander, 1849: 37. TL: Russia [Type: UNK].

Distribution: Makran, Balochistan (Brown, 1959: 229).

\section{Cataglyphis cugiai Menozzi, 1939}

Cataglyphis (Monocombus) cugiai Menozzi, 1939: 323. TL: Suru valley, Kargil, Jammu and Kashmir: India [Holotype: MZPW].

Distribution: Askole, Blukro, Braldu, Bunji, Dassu, Nanga Parbat, Panmah, Skardu, Talichi, Gilgit-Baltistan (Menozzi, 1939: 326; Eidmann, 1942: 255; Mani \& Singh, 1962: 84).

\section{Cataglyphis setipes (Forel, 1894)}

Myrmecocystus viaticus $R$. setipes Forel, 1894: 401. TL: Nusseerabad, Rajpootana: India [Syntype: MHNG].

Distribution: Gor, Gilgit-Baltistan (Eidmann, 1942: 254); Kalat, Makran, Pasni, Quetta, Balochistan; Sindh (Brown, 1959: 229).

\section{Formica clara Forel, 1886}

Formica rufibarbis var. clara Forel, 1886: 206. TL: [Damas] Damascus: Syria [Syntype: MHNG].

Distribution: Pakistan (Seifert \& Schultz, 2009: 263).

\section{Formica cunicularia Latreille, 1798}

Formica cunicularia Latreille, 1798: 40. TL: Fumel: France [Neotype: SMNG].

Distribution: Kashmir (Menozzi, 1939: 343); Bunji, Chamuri, Gilgit-Baltistan (Eidmann, 1942: 252); Chamuri, Nanga Parbat (Mani \& Singh, 1962: 85).

\section{Formica fusca Linnaeus, 1758}

Formica fusca Linnaeus, 1758: 580. TL: Sweden [Type: UNK]. Distribution: Karakorum (Menozzi, 1939: 343).

\section{Formica gagates Latreille, 1798}

Formica gagates Latreille, 1798: 36. TL: Limousin, Brive: France [Neotype: MNHN].

Distribution: Chamuri, Hauptlager, Nanga Parbat (Eidmann, 1942: 253).

\section{Formica pamirica Dlussky, 1965}

Formica subpilosa subsp. pamirica Dlussky, 1965: 30. TL: Darvaz Mountain Ridge, Highway to Horog: Tajikistan [Holotype: ZISP].

Distribution: Alay valley, base of Amu Darya (Dlussky, 1965: 30).

\section{Formica picea Nylander, 1846}

Formica picea Nylander, 1846: 917. TL: Helsinki: Finland [Lectotype: FMNH].

Distribution: Karakorum (Menozzi, 1939: 343); Askole, Baltoro, Braldu, Panmah, Gilgit-Baltistan (Mani \& Singh, 1962: 85). 

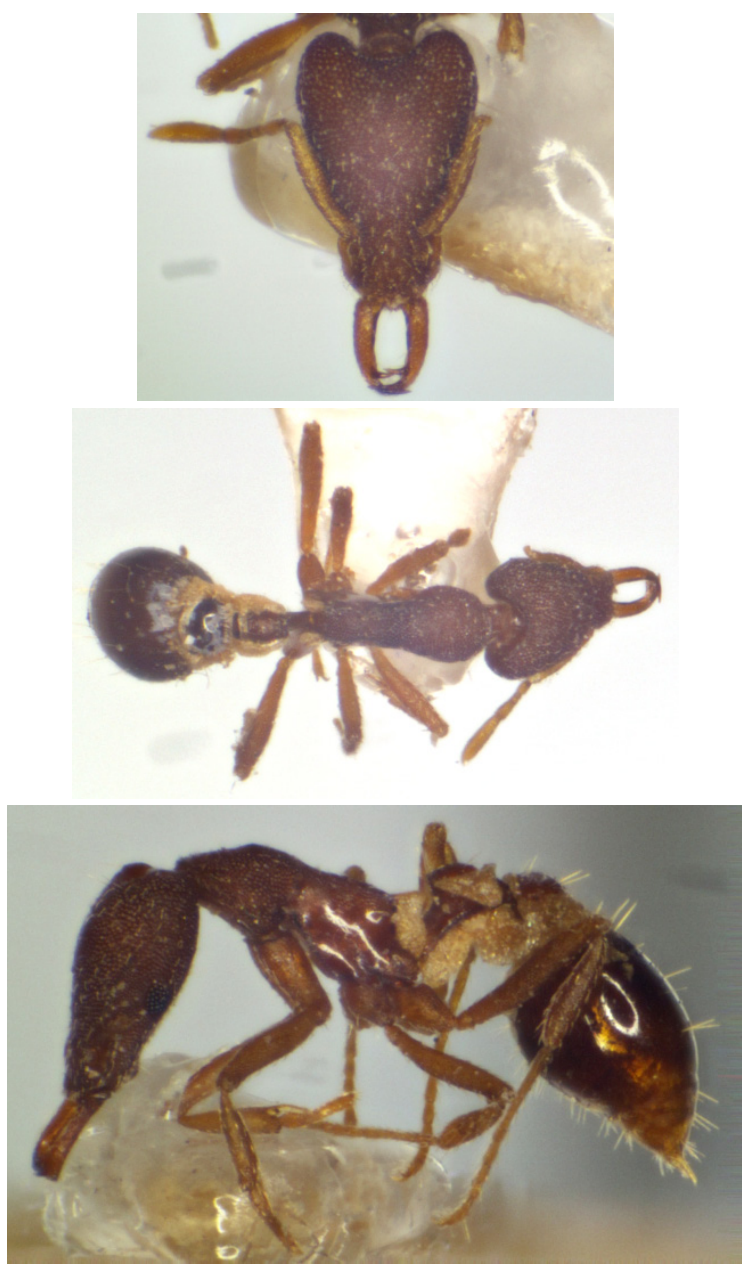

Figures 5-7. Strumigenys godeffroyi Mayr, 1866 (5) Head, frontal view; Habitus (6) dorsal view; (7) lateral view.

\section{Formica sanguinea Latreille, 1798}

Formica sanguinea Latreille, 1798: 37. TL: France [Type: UNK].

Distribution: Karakorum (Menozzi, 1939: 344).

\section{Formica truncorum Fabricius, 1804}

Formica truncorum Fabricius, 1804: 403. TL: Czechoslovakia [Holotype: UNK].

Distribution: Doyan, Gor, Gilgit-Baltistan (Eidmann, 1942: 253).

\section{Lasius alienoflavus Bingham, 1903}

Lasius alienoflavus Bingham, 1903: 341. TL: Himalayas: India [Syntypes: AMNH].

Distribution: Bara Gali, Chikar, Chopal, Kawai Kaghan, Murree, Nathia Gali (Collingwood, 1982: 290).

\section{Lasius alienus (Foerster, 1850)}

Formica aliena Foerster, 1850: 36. TL: Lousberg, Aachen: Germany [Syntype: NHMW].

Distribution: Chokpiong, Dassu, Gund, Gilgit-Baltistan; Quetta, Balochistan (Menozzi, 1939: 312); Naran, Kaghan (Collingwood, 1982: 285); Islamabad, Rawalpindi (Umair et al., 2012: 703).

\section{Lasius brunneus (Latreille, 1798)}

Formica brunnea Latreille, 1798: 41. TL: France. [Type: UNK].
Distribution: Lahore (Wilson, 1955: 51); Kalan, Swat (Collingwood, 1982: 286).

\section{Lasius carniolicus Mayr, 1861}

Lasius carniolicus Mayr, 1861: 51. TL: Laibach: Yugoslavia [Holotype: NHMW].

Distribution: Askole, Braldu, Gilgit-Baltistan (Menozzi, 1939: 313; Wilson, 1955: 191); Naran (Collingwood, 1982: 292).

\section{Lasius himalayanus Bingham, 1903}

Lasius himalayanus Bingham, 1903: 340. TL: Himalaya: India [Syntypes: MHNG].

Distribution: Shigar, Gilgit-Baltistan (Menozzi, 1939: 313); Chitral, Hazara, Lahore (Seifert, 1992: 8).

\section{Lasius hirsutus Seifert, 1992}

Lasius (Lasius) hirsutus Seifert, 1992: 41. TL: Chitral, Madaglasht: Pakistan [Syntypes: MHNG].

Distribution: Chitral, Madaglasht (Seifert, 1992: 41).

\section{Lasius lawarai Seifert, 1992}

Lasius (Lasius) lawarai Seifert, 1992: 38. TL: Dir, LawaraiPass: Pakistan [Holotype: MHNG].

Distribution: Dir, Lawarai-Pass; Chitral, Madaglasht; Hazara, Naran; Swat, Kalam, Malam Jabba, Miandam, Utrot (Seifert, 1992: 38); Lawarai-Pass (CASENT0911182: AntWeb: 2019).

\section{Lasius niger (Linnaeus, 1758)}

Formica nigra Linnaeus, 1758: 580. TL: Europe [Type: UNK].

Distribution: Askole, Baltoro, Biaho, Braldu, Panmah, Payu Urdukas, Tsok, Gilgit-Baltistan (Menozzi, 1939: 313); Nathia Gali (Wilson, 1955: 76); Naran, Kaghan valley (Collingwood, 1982: 287).

\section{Lasius wittmeri Seifert, 1992}

Lasius (Lasius) wittmeri Seifert, 1992: 39. TL: Pahalgam, Jammu and Kashmir: India [Holotype: NHMB].

Distribution: Naran, Kaghan valley (Seifert, 1992: 39).

\section{Lepisiota frauenfeldi (Mayr, 1855)}

Hypoclinea frauenfeldi Mayr, 1855: 378. TL: [Syracus], Sicily: Italy; Attica: Greece [Syntypes: ZMHB].

Distribution: Islamabad, Rawalpindi (Umair et al., 2012: 702).

\section{Lepisiota frauenfeldi integra (Forel, 1894)}

Acantholepis frauenfeldi Var. integra Forel, 1894: 411. TL: Dharamsala, Himachal Pradesh: India [Syntype: MHNG]. Distribution: Talichi, Gilgit-Baltistan (Eidmann, 1942: 249).

\section{Lepisiota lunaris (Emery, 1893)}

Acantholepis lunaris Emery, 1893: 250. TL: Colombo: Sri Lanka [Syntypes: MSNG].

Distribution: Margund, Sindh valley (Menozzi, 1939: 312). 


\section{Lepisiota sericea (Forel, 1892)}

Acantholepis frauenfeldi var. sericea Forel, 1892b: 41. TL:

Mussoorie, Uttarakhand: India [Syntype: MHNG].

Distribution: Dassu, Gangam, Gārhi, Gilgit-Baltistan (Menozzi, 1939: 312); Makran, Nodiz; Sindh, Thatta (Brown, 1959: 229).

\section{Paratrechina longicornis (Latreille, 1802)}

Formica longicornis Latreille, 1802: 113. TL: Bangkok: Thailand [Neotype: ANIC].

Distribution: Pakistan (AntMaps: 2019).

\section{Plagiolepis balestrierii Menozzi, 1939}

Plagiolepis balestrierii Menozzi, 1939: 310. TL: Karakoram: India, Pakistan [Syntypes: MSNM].

Distribution: Askole, Braldo, Chongo, Kro Brok, Parkutta, Shigar, Skardu, Gilgit-Baltistan (Menozzi, 1939: 312; Mani \& Singh, 1962: 85).

\section{Plagiolepis pontii Menozzi, 1939}

Plagiolepis pontii Menozzi, 1939: 309. TL: Panmah valley, Suru valley, Jammu and Kashmir: India [Syntypes: MSNM]. Distribution: Panmah, Gilgit-Baltistan (Menozzi, 1939: 310).

\section{Polyrhachis grisescens Emery, 1895}

Polyrhachis simplex var. grisescens Emery, 1895: 483. TL: Lower Burma, Pegu Yoma: Myanmar [Syntype: MSNG].

Distribution: Kohat (CASENT0217772: AntWeb, 2019).

\section{Polyrhachis hodgsoni Forel, 1902}

Polyrhachis hodgsoni Forel, 1902a: 289. TL: [Moulmain] Mawlamyine: Myanmar [Syntype: MHNG].

Distribution: Islamabad (Umair et al., 2012: 702).

\section{MYRMICINAE}

\section{Aphaenogaster beesoni Donisthorpe, 1933}

Aphaenogaster (Attomyrma) beesoni Donisthorpe, 1933: 24. TL: Kotkhai, Simla, Himachal Pradesh: India [Holotype: BMNH]. Distribution: Kalala, North-West Frontier Province (Donisthorpe, 1933: 24).

\section{Aphaenogaster sagei (Forel, 1902)}

Stenamma (Aphaenogaster) sagei Forel, 1902b: 221. TL: Lahoul front Thibet, Himachal Pradesh: India [Syntype: MHNG]. Distribution: Askole, Gilgit-Baltistan (Menozzi, 1939: 296); Kaghan valley, Khyber Pakhtunkhwa (CASENT0280946: AntWeb, 2019).

\section{Aphaenogaster smythiesii (Forel, 1902)}

Stenamma (Aphaenogaster) smythiesii Forel, 1902b: 222. TL:

Mandali, N. W. Himalaya: India [Syntype: MHNG].

Distribution: Quetta, Balochistan (Menozzi, 1939: 296).

\section{Cardiocondyla mauritanica Forel, 1890}

Cardiocondyla nuda var. mauritanica Forel, 1890a: 1xxv. TL: Gabés, Tunisia [Syntype: MHNG].
Distribution: Madyan, Swat (Seifert, 2003: 249; 2008: 45; Wetterer, 2012: 990; Seifert et al., 2017: 339).

\section{Cardiocondyla wroughtonii (Forel, 1890) Figures (8-10)}

Emeryia wroughtonii Forel, 1890b: cxi. TL: Poona: India [Syntypes: BMNH, MHNG].

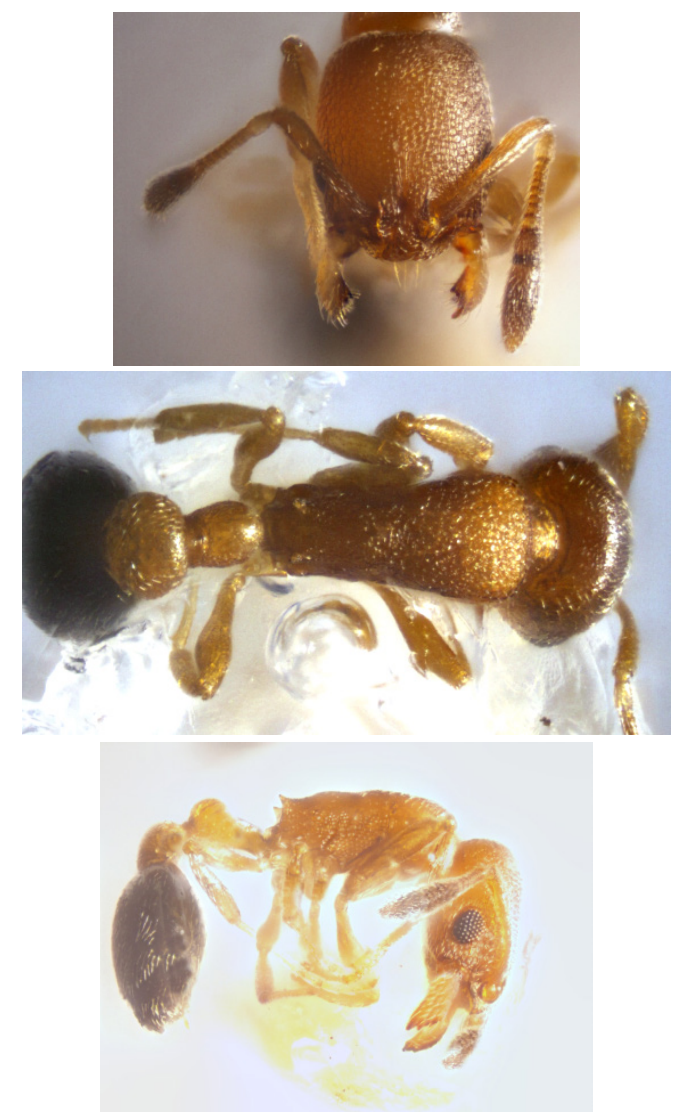

Figures 8-10. Cardiocondyla wroughtonii (Forel, 1890) (8) Head, frontal view; Habitus (9) dorsal view; (10) lateral view.

Material examined: Pakistan, Islamabad, Trail 5 Forest area, $33^{\circ} 45.247^{\prime} \mathrm{N} 73^{\circ} 05.146^{\prime} \mathrm{E}, 631 \mathrm{~m}$. a.s.1., 5 (workers), 20.ii.2015, leaf litter, 5 (workers), 05.iv.2017, soil nest, 11 (workers), 14.x.2017, leaf litter, leg. M.T. Rasheed; Rawalpindi, Forest area Murree $33^{\circ} 51.008^{\prime} \mathrm{N} 73^{\circ} 19.162^{\prime} \mathrm{E}$, $1158 \mathrm{~m}$ a.s.1., 4 (workers), 20.iv.2015, flowering plants, leg. I. Bodlah; Rawalpindi, Forest area Kotli Sattian, $33^{\circ} 41.902^{\prime} \mathrm{N}$ 07330.612'E, $1261 \mathrm{~m}$ a.s.1., 9 (workers), 05.xi.2016, leaf litter, leg. I. Bodlah; Rawalpindi, Forest area Osia, $33^{\circ} 43.255^{\prime} \mathrm{N} 073^{\circ} 02.150^{\prime} \mathrm{E}, 1482 \mathrm{~m}$ a.s.l., 6 (workers), 19.x.2016, leaf litter, leg. M.T. Rasheed; Islamabad, Forest area Pir Sohawa, $33^{\circ} 78.82^{\prime} \mathrm{N} 73^{\circ} 10.69^{\prime} \mathrm{E}, 1482 \mathrm{~m}$ a.s.l., 12 (workers), 23.viii.2016, nursery plants, leg. I. Bodlah; Rawalpindi, Forest area Neela Sand, $33^{\circ} 95.16^{\prime} \mathrm{N} 73^{\circ} 22.007^{\prime} \mathrm{E}$, 923 m a.s.1., 10 (workers), 04.vi.2017, soil nest, leg. A. G. Fareen; Rawalpindi, Forest area Neela Sand, $33^{\circ} 51.008^{\prime} \mathrm{N}$ 73¹9.162'E, $1482 \mathrm{~m}$ a.s.1., 10 (workers), 25.ix.2017, soil nest, leg. A. G. Fareen; Islamabad, Kachnar Park Forest area, $33^{\circ} 40.602^{\prime} \mathrm{N} 73^{\circ} 04.574^{\prime} \mathrm{E}, 603 \mathrm{~m}$. a.s.l., 1 (worker), 31.viii.2017, tree bark, leg. M.T. Rasheed.

Distribution: Pakistan (new record). 


\section{Crematogaster biroi Mayr, 1897 Figures (11-13)}

Crematogaster biroi Mayr, 1897: 428. TL: Colombo [Ceylon]: Sri Lanka [Syntype: HNHM].

Material examined. Pakistan, Islamabad, Forest area

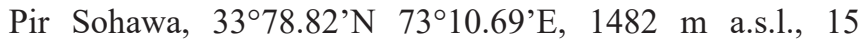
(workers), 11.iv.2016, tree bark, leg. I. Bodlah; Rawalpindi, Forest area Neela Sand, 339.516’N 73²2.007'E, 923 m a.s.1., 5 (workers), 09.v.2016, tree bark, leg. A. G. Fareen; Rawalpindi, Forest area Murree 3351.008 N' $73^{\circ} 19.162$ E', 1158 m a.s.1., 12 (workers), 08.vi.2015, tree bark, leg. A. G. Fareen; Islamabad, Kachnar Park Forest area, $33^{\circ} 40.602^{\prime} \mathrm{N}$ 7304.574'E, 603 m. a.s.1., 10 (workers), 26.iii.2017, soil nest, leg. M.T. Rasheed.

Distribution: Pakistan (new record).

\section{Crematogaster himalayana Forel, 1902}

Crematogaster himalayanus Forel, 1902b: 202. TL: Dharamsala, Himachal Pradesh: India [Syntype: MHNG].

Distribution: Rakhiot-Tal, Nanga Parbat (Eidmann, 1942: 247).

\section{Crematogaster rothneyi Mayr, 1879}

Crematogaster rothneyi Mayr, 1879: 685. TL: Calcutta, West Bengal: India [Syntype: NHMW].

Distribution: Islamabad, Rawalpindi (Umair et al., 2012: 702).

\section{Crematogaster subdentata kaschgariensis Forel, 1901}

Crematogaster inermis R. kaschgariensis Forel, 1901b: 63. TL: Maralbaschi am Kaschgar-Darja: China [Syntype: MHNG].

Distribution: Kashmir (Menozzi, 1939: 335).

Leptothorax acervorum (Fabricius, 1793)

Formica acervorum Fabricius, 1793: 358. TL: Denmark [Type: UNK].

Distribution: Chogo, Thla Brok, Urdukas, Gilgit-Baltistan (Menozzi, 1939: 302).

\section{Meranoplus bicolor (Guérin-Méneville, 1844)}

Cryptocerus bicolor Guérin-Méneville, 1844: 425. TL: Pudicherry, Tamil Nadu: India [Type: UNK].

Distribution: Kohat, Rawalpindi (Schödl, 1998: 372); Islamabad, Rawalpindi, (Umair et al., 2012: 701); Kohat (CASENT0217854: AntWeb, 2019).

\section{Messor himalayanus (Forel, 1902)}

Stenamma (Messor) barbarum r. himalayanum Forel, 1902b: 221. TL: Jammu and Kashmir: India Syntypes: MHNG].

Distribution: Chongo, Gārhi, Gund, Hoto, Karal Marfo, Gilgit-Baltistan; Quetta, Balochistan (Menozzi, 1939: 297); Turbaling, Gilgit-Baltistan (Eidmann, 1942: 245).

\section{Messor instabilis (Smith, 1858)}

Atta instabilis Smith, 1858: 163. TL: N. India: India [Syntype: $\mathrm{BMNH}]$.

Distribution: Gārhi, Skardu, Gilgit-Baltistan (Menozzi, 1939:

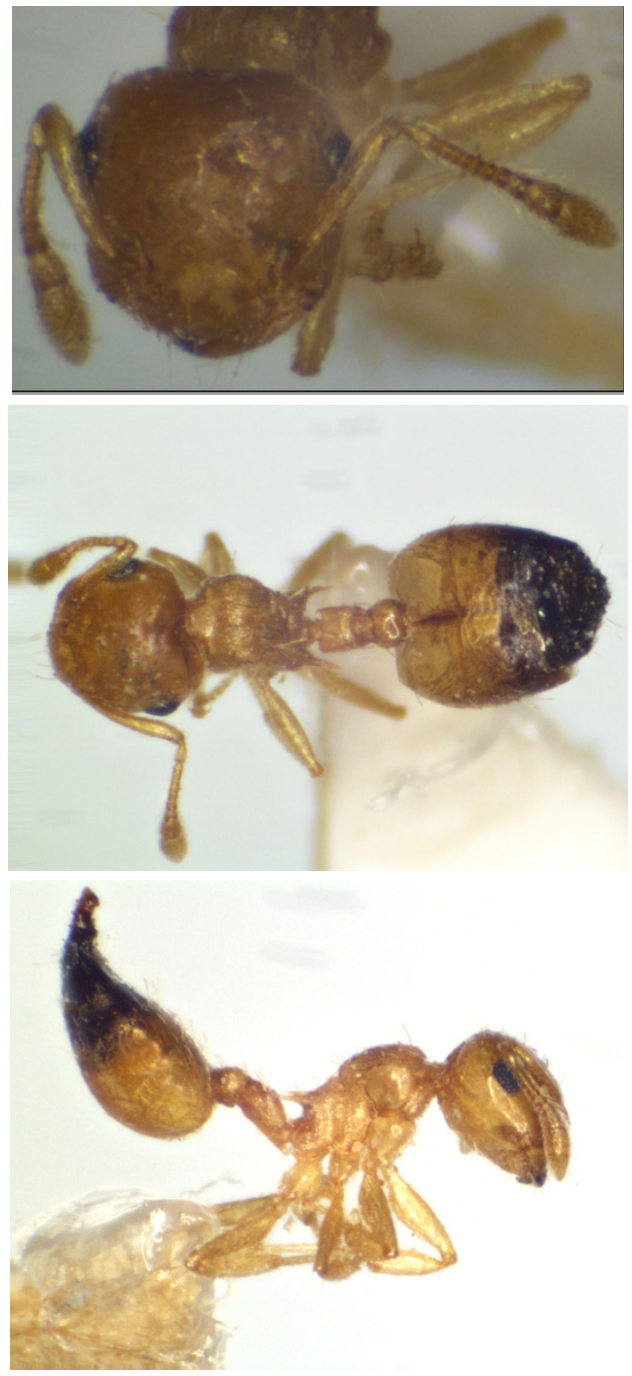

Figures 11-13. Crematogaster biroi Mayr, 1897 (11) Head, frontal view; Habitus (12) dorsal view; (13) lateral view.

297); Astore, Bunji, Gilgit-Baltistan (Eidmann, 1942: 246). Messor reticuliventris Karavaiev, 1911

Messor barbarus subsp. reticuliventris Karavaiev, 1911: 67. TL: Firusa: Turkmenistan [Syntype: MHNG].

Distribution: Quetta, Balochistan (CASENT0281594: AntWeb, 2019).

\section{Monomorium indicum Forel, 1902}

Monomorium salomonis r. indicum Forel, 1902b: 213. TL: Tiruchirappalli, Tamil Nadu: India [Syntype: HNHM].

Distribution: Kalat, Makran, Nodiz, Balochistan; Thatta, Sindh (Brown, 1959: 229).

\section{Monomorium longi Forel, 1902}

Monomorium longi Forel, 1902b: 211. TL: Garo Hills, Meghalaya: India [Syntype: MHNG].

Distribution: Islamabad (Umair et al., 2012: 701).

\section{Monomorium luisae Forel, 1904}

Monomorium luisae Forel, 1904: 25. TL: Jhelum valley, Jammu and Kashmir: India [Syntype: MHNG]. 
Distribution: Kashmir (Forel, 1904: 25; Menozzi, 1939: 336).

\section{Monomorium sagei Forel, 1902}

Monomorium sagei Forel, 1902b: 211. TL: Dharamsala, Himachal Pradesh: India [Syntype: MHNG].

Distribution: Karakorum (Menozzi, 1939: 336); Baltistan (Pisarski, 1967: 395).

\section{Monomorium schurri Forel, 1902}

Monomorium schurri Forel, 1902b: 212. TL: Ootacamund, Nilgiris, Tamil Nadu: India [Syntypes: BMNH, MHNG].

Distribution: Islamabad, Rawalpindi, (Umair et al., 2012: 701).

\section{Myrmica aimonissabaudiae Menozzi, 1939}

Myrmica aimonissabaudiae Menozzi, 1939: 286. TL: Karakorum, Gund (Sindh): Pakistan [Lectotype: MSNM].

Distribution: Askole, Dassu, Doyan, Gol, Gund, Kushumul, Olthingthang, Parkutta, Shigar, Skardu, Tolti, Gilgit-Baltistan; Quetta, Balochistan (Menozzi, 1939: 289; Eidmann, 1942: 245; Mani \& Singh, 1962: 85); Karakorum (Weber, 1947: 464); Shogran, Kaghan valley; Kalam, Changla Gali, Madaglasht, Naran (Radchenko \& Elmes, 2001: 249; Bharti et al., 2016: 963); Gund (Sindh) (CASENT0904554: AntWeb, 2019).

\section{Myrmica brancuccii Radchenko, Elmes and Collingwood, 1999} Myrmica brancuccii Radchenko, Elmes and Collingwood, 1999: 30 in Radchenko and Elmes, 1999. TL: Utrot: Pakistan [Holotype: BMNH].

Distribution: Chitral, Lawarai, Utrot (Radchenko \& Elmes, 1999: 30; 2001: 260; 2010: 106; Bharti et al., 2016: 964); Utrot (CASENT0900337: AntWeb: 2019).

\section{Myrmica cachmiriensis Forel, 1904}

Myrmica smythiesii r. cachmiriensis Forel, 1904: 23. TL: Sindh valley, Jammu and Kashmir: India [Holotype: MHNG]. Distribution: Askole, Chokpiong, Hoto, Shimtsa, Tsok, GilgitBaltistan (Menozzi, 1939: 292; Mani \& Singh, 1962: 85); Changla Gali, Sobodan Gali (Bagh), Shogran, Kaghan valley (Radchenko \& Elmes, 2001: 251, 2010: 108; Bharti et al., 2016: 964).

\section{Myrmica ordinaria Radchenko and Elmes, 1999}

Myrmica ordinaria Radchenko \& Elmes, 1999: 41. TL: Seven Spring, Pahalgam, Jammu and Kashmir: India [Holotype: BMNH].

Distribution: Kalam (Radchenko \& Elmes, 1999: 41; 2001: 252; 2010: 209; Bharti et al. 2016: 967).

\section{Myrmica pseudorugosa Bharti, 2012}

Myrmica pseudorugosa Bharti, 2012: 12. TL: Kaghan valley, Gittidas: Pakistan [Holotype: PUAC].

Distribution: Gittidas, Kaghan valley (Bharti, 2012: 12; Bharti et al., 2016: 967; ANTWEB1008037: AntWeb, 2019).

\section{Myrmica rigatoi Radchenko and Elmes, 1998}

Myrmica rigatoi Radchenko and Elmes, 1998: 18. TL: Changla Gali: Pakistan [Holotype: MSNM].
Distribution: Changla Gali, Hazara Durgo Gal (Radchenko \& Elmes, 1998: 18; 1999: 41; 2001: 256; 2010: 225; Bharti et al., 2016: 968; CASENT0904551: AntWeb, 2019).

\section{Myrmica vittata Radchenko and Elmes, 1999}

Myrmica vittata Radchenko and Elmes, 1999: 33. TL: Bumburet: Pakistan [Holotype: BMNH].

Distribution: Bumburet, Kalam (Radchenko \& Elmes, 1999: 33; 2001: 260; 2010: 319; Bharti et al., 2016: 970; CASENT0900334: AntWeb, 2019).

\section{Myrmica wardi Radchenko and Elmes, 1999}

Myrmica wardi Radchenko and Elmes, 1999: 38. TL: Leh, Jammu and Kashmir: India [Holotype: BMNH].

Distribution: Chitral valley (Radchenko \& Elmes, 1999: 38; 2001: 252; 2010: 320; Bharti et al., 2016: 971).

\section{Myrmica wittmeri Radchenko and Elmes, 1999}

Myrmica wittmeri Radchenko and Elmes, 1999: 38. TL: Mahri, Himachal Pradesh: India [Holotype: NHMB].

Distribution: Kalam (Radchenko \& Elmes, 1999: 38; 2001: 255; 2010: 326; Bharti et al., 2016: 971).

\section{Myrmicaria brunnea Saunders, 1842}

Myrmicaria brunnea Saunders, 1842: 57. TL: India [Type: UNK]. Distribution: Marghalla Hills, Islamabad (Yahya et al., 2009: 251).

\section{Paratopula ceylonica (Emery, 1901)}

Atopomyrmex ceylonicus Emery, 1901: 114. TL: Negombo: Sri Lanka [Syntypes: DEIC, MSNG].

Distribution: Rawalpindi (Umair et al., 2012: 701).

\section{Pheidole indica Mayr, 1879}

Pheidole indica Mayr, 1879: 679. TL: Calcutta, West Bengal: India [Lectotype: NHMW].

Distribution: Gārhi, Gilgit-Baltistan (Menozzi, 1939: 286); Torkham, Peshawar (Pisarski, 1967: 386).

\section{Pheidole mus Forel, 1902}

Pheidole mus Forel, 1902b: 174. TL: Kanara, Karnataka: India [Syntypes: MHNG].

Distribution: Islamabad, Rawalpindi (Umair et al., 2012: 701).

\section{Pheidole nietneri Emery, 1901}

Pheidole nietneri Emery, 1901: 118. TL: Bandarawela, Sri Lanka [Syntypes: UNK].

Distribution: Islamabad, Rawalpindi (Umair et al., 2012: 700).

\section{Pheidole pronotalis Forel, 1902}

Pheidole pronotalis Forel, 1902b: 173. TL: Sri Lanka [Syntypes: MHNG].

Distribution: Islamabad, Rawalpindi (Umair et al., 2012: 701).

Pheidole roberti Forel, 1902

Pheidole roberti Forel, 1902b: 183. TL: Kanara, Karnataka: India [Syntypes: MHNG]. 
Distribution: Bunji, Gilgit-Baltistan (Eidmann, 1942: 247).

Pheidole sagei Forel, 1902

Pheidole sagei Forel, 1902b: 174. TL: Dharamsala, Himachal

Pradesh: India [Syntypes: MHNG, MSNG].

Distribution: Gārhi, Gilgit-Baltistan (Menozzi, 1939: 297).

Pheidole singaporensis Özdikmen, 2010

Pheidole singaporensis Özdikmen, 2010: 804. TL: Singapore

[Syntypes: BMNH, OUMNH].

Distribution: Pakistan (Li-zhong, 2006: 263).

\section{Stenamma jeriorum DuBois, 1998}

Stenamma jeriorum DuBois, 1998: 248. TL: [Malan Jabba] Malam Jabba: Pakistan [Holotype: BMNH].

Distribution: Malam Jabba (DuBois, 1998: 250; Liu \& Xu, 2011: 737; CASENT0900947: AntWeb, 2019).

\section{Stenamma kashmirense Baroni Urbani, 1977}

Stenamma kashmirense Baroni Urbani, 1977: 415. TL: Yusmarg, Jammu and Kashmir: India [Holotype: NHMB].

Distribution: Naran, Kaghan valley (DuBois, 1998: 248; Liu \& Xu, 2011: 737; CASENT0900947: AntWeb, 2019).

\section{Strumigenys godeffroyi Mayr, $1866 \quad$ Figures (5-7)}

Strumigenys godeffroyi Mayr, 1866b: 516. TL: Samoa [Syntype: NHMW].

Material examined. Pakistan, Rawalpindi, Forest area Kotlisatian, $33^{\circ} 41.902^{\prime} \mathrm{N} 73^{\circ} 30.612^{\prime} \mathrm{E}, 1261$ m. a.s.l., 25 (workers), 12.iv.2016, 50 (workers), 21.vi.2017, under stone, leg. A. G. Fareen.

Distribution: Pakistan (new record).

\section{Temnothorax desioi (Menozzi, 1939)}

Leptothorax (Leptothorax) desioi Menozzi, 1939: 303. TL: Karakorum, Askole (Braldu): Pakistan [Syntype: MSNM].

Distribution: Askole, Baltoro, Bolta, Boorgi Nullah, Dumultar, Panmah valley, Payu, Karal Marfo, Shiniltalmosa, Tsok, GilgitBaltistan (Menozzi, 1939: 297; CASENT0904558: AntWeb, 2019); Turbaling, Gilgit-Baltistan (Eidmann, 1942: 245).

\section{Temnothorax pamiricus (Ruzsky, 1902)}

Leptothorax pamiricus Ruzsky, 1902: 478. TL: Pamir: Russia [Lectotype: MSNG].

Distribution: Askole, Bardumal, Biaho, Burdwan, Chongo, Hoto, Korophon, Kro Brok, Payu, Gilgit-Baltistan (Menozzi, 1939: 303).

\section{Tetramorium nursei Bingham, 1903}

Tetramorium nursei Bingham, 1903: 181. TL: Quetta, N.W. Frontier: Pakistan [Syntype: BMNH].

Distribution: Quetta, Balochistan (Bingham, 1903: 181; Bolton, 1977: 93; Li-zhong, 2006: 273; CASENT0901103: AntWeb, 2019).

\section{Tetramorium salvatum Forel, 1902}

Tetramorium salvatum Forel, 1902b: 235. TL: [N. Indien], India [Syntype: MHNG].
Distribution: Kalash (CASENT0280890: AntWeb, 2019).

\section{Tetramorium smithi Mayr, 1879}

Tetramorium smithi Mayr, 1879: 673. TL: Calcutta, West Bengal: India [Syntypes: BMNH, NHMW].

Distribution: Islamabad, Rawalpindi (Umair et al., 2012: 702).

\section{Tetramorium sulcinode Santschi, 1927}

Tetramorium caespitum var. sulcinode Santschi, 1927: 53. TL: [Ssemiretschie, Ssukuluk Est de Pichpek], Kazakhstan [Lectotype: NHMB].

Distribution: Sirani (Csösz et al., 2007: 33).

\section{Trichomyrmex glaber (André, 1883)}

Holcomyrmex glaber André, 1883: 345. TL: India [Syntype: MNHN].

Distribution: Rawalpindi (Umair et al., 2012: 700).

\section{Trichomyrmex scabriceps (Mayr, 1879)}

Holcomyrmex scabriceps Mayr, 1879: 672. TL: Calcutta, West Bengal: India [Syntypes: BMNH, NHMW].

Distribution: Rawalpindi (Umair et al., 2012: 700).

\section{PONERINAE}

\section{Diacamma scalpratum (Smith, 1858)}

Ponera scalprata Smith, 1858: 84. TL: N. India: India [Lectotype: NHMW].

Distribution: Sindh (Laciny etal., 2015: 90; CASENT0915970:

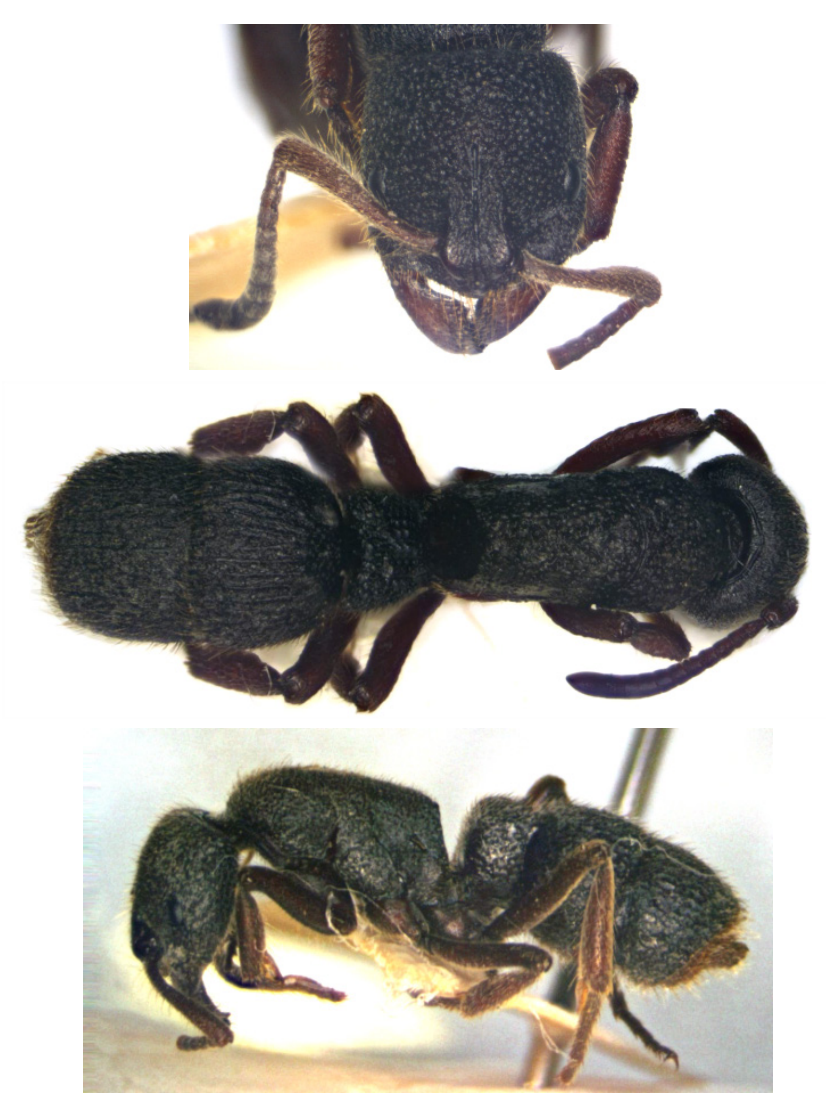

Figures 14-16. Pseudoneoponera rufipes (Jerdon, 1851) (14) Head, frontal view; Habitus (15) dorsal view; (16) lateral view. 
AntWeb, 2019).

\section{Leptogenys hysterica Forel, 1900}

Leptogenys hysterica Forel, 1900: 311. TL: Belgaum: India [Syntype: NHMB].

Distribution: Islamabad, Rawalpindi (Rasheed et al., 2018: 13033).

Odontoponera denticulata (Smith, 1858)

Ponera denticulata Smith, 1858: 90. TL: Singapore [Holotype: BMNH].

Distribution: Pakistan (Yamane, 2009: 7).

Pseudoneoponera rufipes (Jerdon, 1851) Figures (14-16)

Ponera rufipes Jerdon, 1851: 119. TL: Malabar, Kerala: India [Type: Lost].

Material examined. Pakistan, Rawalpindi, Forest area Kotlisatian, $33^{\circ} 41.902^{\prime} \mathrm{N} \quad 073^{\circ} 30.612^{\prime} \mathrm{E}, 1261$ m. a.s.l., 3 (workers), 31.vii.2016, under stone, leg. A. G. Fareen; Islamabad, Trail 5 Forest area, $36^{\circ} 14.359^{\prime} \mathrm{N} 74^{\circ} 30.037^{\prime} \mathrm{E}$, 639 m. a.s.1., 5 (workers), 02.iii.2017, 6 (workers), 15.iv.2017, under stone, leg. M.T. Rasheed; Rawalpindi, Forest area

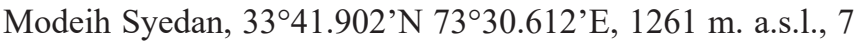
(workers), 09.iv.2016, leg. A. G. Fareen; Islamabad, Forest area Pir Sohawa, $33^{\circ} 78.82^{\prime} \mathrm{N} 73^{\circ} 10.69^{\prime} \mathrm{E}, 1047$ m. a.s.l., 4 (workers), 09.v.2017, under stone, leg. A. G. Fareen.

Distribution: Pakistan (new record).

\section{PROCERATIINAE}

\section{Proceratium confinium de Andrade, 2003}

Proceratium confinium de Andrade, 2003: 248 in Baroni Urbani and de Andrade, 2003. TL: Surat, Malkandi: Pakistan [Holotype: MHNG].

Distribution: Malkandi Kaghan (Baroni Urbani \& de Andrade, 2003: 249; CASENT0911253: AntWeb, 2019).

\section{PSEUDOMYRMECINAE}

\section{Tetraponera allaborans (Walker, 1859)}

Pseudomyrma allaborans Walker, 1859: 375. TL: Sri Lanka [Syntypes: BMNH].

Distribution: Islamabad, Rawalpindi (Bodlah et al., 2016: 1029).

\section{Tetraponera nigra (Jerdon, 1851)}

Eciton nigrum Jerdon, 1851: 112. TL: Malabar, Kerala: India [Type: Lost].

Distribution: Sindh (Ward, 2001: 635); Islamabad, Rawalpindi (Bodlah et al., 2016: 1029).

\section{Tetraponera rufonigra (Jerdon, 1851)}

Eciton rufonigrum Jerdon, 1851: 111. TL: Malabar, Kerala: India [Type: Lost].

Distribution: Lahore (Ward, 2001: 650; CASENT0752675:

AntWeb, 2019).

\section{Discussion}

A total of 103 species of ant, belonging to 35 genera in seven subfamilies are known to occur across vast areas of Pakistan. The seven subfamilies include: Dolichoderinae, Dorylinae, Formicinae, Myrmicinae, Ponerinae, Proceratiinae and Pseudomyrmecinae. Among these, Myrmicinae is most diversity with 15 genera and 48 species followed by Formicinae with 8 genera and 39 species; Dorylinae 4 genera and 5 species; Ponerinae 4 genera and 4 species; Dolichoderinae 2 genera and 3 species; while Proceratiinae and Pseudomyrmecinae are represented by single genus with one and 3 species respectively. The most diverse genera in terms of species are Camponotus (10), Lasius (9), Myrmica (9), Formica (8), Pheidole (7), Temnothorax (6), Monomorium (5), followed by others. Two subfamilies; Formicinae and Myrmicinae together contribute more about $85 \%$ to the regional ants. Like rest of the flora and fauna of the country (GOP, 1999), the rate of endemism in ants is also very low. Of the known species from the region only seven species are considered to be endemic. These include: Lasius hirsutus Seifert, 1992; Lasius lawarai Seifert, 1992; Myrmica pseudorugosa Bharti, 2012; Myrmica rigatoi Radchenko and Elmes, 1998; Myrmica vittata Radchenko and Elmes, 1999; Stenamma jeriorum DuBois, 1998 and Proceratium confinium de Andrade, 2003.

The species list generated here cannot be complete, as the widespread ants present in neighboring countries of Pakistan with similar climatic conditions should be there. However, we hope that it will facilitate future discovery, and conservation of ants in Pakistan.

\section{Acknowledgments}

We are grateful to Pakistan Science foundation for funding under the project PSF/NSLP/P-UAAR (313) for these findings. We are highly appreciative to AntMaps (www. antmaps.org), AntWeb (www.antweb.org) and AntWiki (www. antwiki.org) for their outstanding work on these entities.

\section{Author Contributions}

Muhammad Tariq Rasheed - Survey, collection and identification.

Imran Bodlah - Survey, collection, identification and data handling.

Ammara Gull e Fareen - Survey, collection and identification. Aijaz Ahmad Wachkoo - Data mining, manuscript write up and species confirmation.

Xiaolei Huang - Data analysis, macrophotography, manuscript review and editing.

Shahid Ali Akbar - Data handling and manuscript write up.

\section{References}

Andersen, A.N. \& Majer, J. D. (2004). Ants show the way Down 
Under: invertebrates as bioindicators in land management. Frontiers in Ecology and the Environment, 2: 291-298. doi: 10.1890/1540-9295(2004)002[0292:ASTWDU]2.0.CO;2

Andersen, A.N., Hertog, T. \& Woinarski, J.C.Z. (2006). Long-term fire exclusion and ant community structure in an Australian tropical savanna: congruence with vegetation succession. Journal of Biogeogrphy, 33: 823-832. doi: 10.11 11/j.1365-2699.2006.01463.x

André, E. (1883). Les fourmis. Species des Hyménoptères d'Europe et d'Algérie. Beaune: Tome Deuxième, 919+48 p

AntMaps, (2019). Global Ant Biodiversity Informatics. http:// antmaps.org/about.html (accessed date: 8 May, 2019).

AntWeb, (2019). Bolton World Catalog. https://www.antweb. org/project.do?name=worldants. (accessed date: 8 May, 2019).

Anwar, M. \& Shank, C.C. (2002). Pakistan. In J.C. Reid (Ed.), Biodiversity planning in Asia - a review of national biodiversity strategies and action plans (NBSAPs) (pp. 430447). Colombo: IUCN Regional Biodiversity Programme Asia.

Aslam, M., Shaheen, F.A. \& Ayyaz, A. (2006). Management of Callosobruchus chinensis Linnaeus in stored chickpea through interspecific and intraspecific predation by ants. World Journal of Agricultural Sciences, 2: 85-89.

Baig, M.B. \& Al-Subaiee, F.S. (2011). Biodiversity in Pakistan: key issues. Biodiversity, 10: 20-29. doi: 10.1080/ 14888386.2009.9712858

Baroni Urbani, C. \& de Andrade, M.L. (2003). The ant genus Proceratium in the extant and fossil record (Hymenoptera: Formicidae). Museo Regionale di Scienze Naturali, 36: 1-492.

Baroni Urbani, C. (1977). Ergebnisse der Bhutan-Expedition 1972 des Naturhistorischen Museums in Basel. Hymenoptera: Fam. Formicidae Genus Stenamma, con una nuova specie del Kashmir. Entomologica Basiliensa, 2: 415-422.

Bharti, H. (2012). Two new species of the genus Myrmica (Hymenoptera: Formicidae: Myrmicinae) from the Himalaya. Tijdschrift voor Entomologie, 155: 9-14. doi: 10.1163/00 4074912 X631742

Bharti, H., Sasi, S. \& Radchenko, A. (2016). Biogeography and Ecology of Myrmica species (Formicidae: Myrmicinae) in Himalayan regions. Sociobiology, 63: 956-975. doi: 10.13 102/sociobiology.v63i3.1145

Bingham, C. T. (1903). The fauna of British India, including Ceylon and Burma. Hymenoptera, Vol. II. Ants and Cuckoowasps. London: Taylor and Francis, $506 \mathrm{p}$

Bodlah, I., Bodlah, M.A., Rasheed, M.T., Akhter, T., Aihetasham, A. \& Yousaf, M. (2017a). New distributional records of psyllid, Trioza fletcheri minor Crawford, 1912 and record of its first association with two ant's species in Pothwar. Asian Journal of Agriculture and Biology, 5: 1-6.
Bodlah, I., Rasheed, M.T. \& Bodlah, M.A. (2017b). New distributional records of Tetraponera rufonigra (Jerdon) from Gilgit Baltistan, Pakistan. Asian Journal of Agriculture and Biology, 5: 56-59.

Bodlah, I., Rasheed, M.T., Fareen, A.G., Ajmal, M.S. \& Bodlah, M.A. (2016). First record of two new species of genus Tetraponera (Hymenoptera: Pseudomyrmecinae: Formicidae) from Pakistan. Journal of Entomology and Zoology Studies, 4: 1028-1030.

Bolton, B. (1977). The ant tribe Tetramoriini (Hymenoptera: Formicidae). The genus Tetramorium Mayr in the Oriental and Indo-Australian regions, and in Australia. Bulletin of the British Museum (Natural History), 36: 67-151.

Brown, W.L.Jr. (1959). Appendix G. Insecta collected by the expedition. In H. Field (Ed.), An anthropological reconnaissance in West Pakistan, 1955, with appendixes on the archaeology and natural history of Baluchistan and Bahawalpur (pp. 229-230). Papers of the Peabody Museum of Archaeology and Ethnology: Harvard University.

Collingwood, C.A. (1982). Himalayan ants of the genus Lasius (Hymenoptera: Formicidae). Systematic Entomology, 7: 283-296.

Cox, C.B. \& Moore, P.D. (1993). Biogeography: an ecological and evolutionary approach $\left(5^{\text {th }}\right.$ edn.). Oxford: Blackwell Scientific Publications, $326 \mathrm{p}$

Csösz, S., Radchenko, A. \& Schulz, A. (2007). Taxonomic revision of the Palaearctic Tetramorium chefketi species complex (Hymenoptera: Formicidae). Zootaxa, 1405: 1-38.

Dalla Torre, K. W. von. (1893). Catalogus Hymenopterorum hucusque descriptorum systematicus et synonymicus. Vol. 7. Formicidae (Heterogyna). Leipzig: W. Engelmann, 289 p

Del Toro, I., Ribbons, R.R. \& Pelini, S. L. (2012). The little things that run the world revisited: a review of antmediated ecosystem services and disservices (Hymenoptera: Formicidae), Myrmecological News, 17: 133-146.

Dlussky, G.M. (1965). Ants of the genus Formica L. of Mongolia and northeast Tibet (Hymenoptera, Formicidae). Annals of Zoology (Warsaw), 23: 15-43.

Donisthorpe, H. (1933). A new species of Aphaenogaster (Hym. Formicidae) from India. Stylops, 2: 24.

DuBois, M.B. (1998). A revision of the ant genus Stenamma in the Palaearctic and Oriental regions (Hymenoptera: Formicidae: Myrmicinae). Sociobiology, 29: 193-403.

Eidmann, H. (1942). Zur Kenntnis der Ameisenfauna des Nanga Parbat. Zoologische Jahrbücher. Abteilung für Systematik, Geographie und Biologie der Tiere, 75: 239-266.

Emery, C. (1893). Voyage de M.E. Simon à l'île de Ceylan (janvier-février 1892). Formicides. Entomological Society of America, 62: 239-258. 
Emery, C. (1895). Viaggio di Leonardo Fea in Birmania e regioni vicine. LXIII. Formiche di Birmania del Tenasserim e dei Monti Carin raccolte da L. Fea. Parte II. Annali del Museo Civico di Storia Naturale Giacomo Doria (Genova), 2: 450-483.

Emery, C. (1901). Ameisen gesammelt in Ceylon von Dr. W. Horn 1899. Deutsche Entomologische Zeitschrift, 1901: 113-122.

Emery, C. (1925). Hymenoptera. Fam. Formicidae. Subfam. Formicinae. Genera Insectorum, 183: 1-302.

Fabricius, J.C. (1787). Mantissa insectorum sistens eorum species nuper detectas adiectis characteribus, genericis, differentiis, specificis, emendationibus, observationibus. Tome I. Hafniae [= Copenhagen]: C. G. Proft, 348 p

Fabricius, J.C. (1793). Entomologia systematica emendata et aucta. Secundum classes, ordines, genera, species, adjectis synonimis, locis observationibus, descriptionibus. Tome 2. Hafniae [= Copenhagen]: C. G. Proft, 519 p

Fabricius, J.C. (1798). Supplementum entomologiae systematicae. Hafniae [= Copenhagen]: Proft and Storch, $572 \mathrm{p}$

Fabricius, J.C. (1804). Systema Piezatorum secundum ordines, genera, species, adjectis synonymis, locis, observationibus, descriptionibus. Brunswick: C. Reichard, xiv $+15-439+30 p$

Foerster, A. (1850). Hymenopterologische Studien. 1. Formicariae. Aachen: Ernst Ter Meer, 74 p

Folgarait, P.J. (1998). Ant biodiversity and its relationship to ecosystem functioning: a review. Biodiversity and Conservation, 7: 1221-1244. doi: 10.1023/A:1008891901953

Forel, A. (1886). Études myrmécologiques en 1886. Annales de la Société Entomologique de Belgique, 30: 131-215.

Forel, A. (1890a). Fourmis de Tunisie et de l'Algérie orientale. Annales de la Société Entomologique de Belgique, 34: 61-76.

Forel, A. (1890b). Aenictus-Typhlatta découverte de M. Wroughton. Nouveaux genres de Formicides. Annales de la Société Entomologique de Belgique, 34: 102-114.

Forel, A. (1892a). Les Formicides de l'Empire des Indes et de Ceylan. Part I. Journal of the Bombay Natural History Society, 7: 219-245.

Forel, A. (1892b). Notes myrmécologiques. Annales de la Société Entomologique de Belgique, 36: 38-43.

Forel, A. (1894). Les Formicides de l'Empire des Indes et de Ceylan. Part IV. Journal of the Bombay Natural History Society, 8: 396-420.

Forel, A. (1895). Les Formicides de l'Empire des Indes et de Ceylan. Part V. Journal of the Bombay Natural History Society, 9: 453-472.

Forel, A. (1900). Les Formicides de l'Empire des Indes et de Ceylan. Part VII. Journal of the Bombay Natural History Society, 13: 303-332.
Forel, A. (1901a). Les Formicides de l'Empire des Indes et de Ceylan. Part VIII. Journal of the Bombay Natural History Society, 13: 462-477.

Forel, A. (1901b). Formiciden des Naturhistorischen Museums zu Hamburg. Neue Calyptomyrmex-, Dacryon-, Podomyrmaund Echinopla-Arten. Mitteilungen aus dem Naturhistorischen Museum in Hamburg, 18: 43-82.

Forel, A. (1902a). Variétés myrmécologiques. Annales de la Société Entomologique de Belgique, 46: 284-296.

Forel, A. (1902b). Myrmicinae nouveaux de l'Inde et de Ceylan. Revue Suisse de Zoologie, 10: 165-249.

Forel, A. (1904). Miscellanea myrmécologiques. Revue Suisse de Zoologie, 12: 1-52.

Forel, A. (1907). Formicides du Musée National Hongrois. Annales Historico-Natureles Musei Nationalis Hungarici, 5: $1-42$.

Gibb, H., Sanders, N.J., Dunn, R.R., Watson, S., Photakis, M., Abril, S., Andersen, A.N., Angulo, E., Armbrecht, I., Arnan, X., Baccaro, F.B. Bishop, T.R., Boulay, R., Castracani, C., Del Toro, I., Delsinne, T., Diaz, M., Donoso, D. A., Enríquez, M. L., Fayle, T.M., Feener, D.H., Fitzpatrick, M.C., Gómez, C., Grasso, D.A., Groc, S., Heterick, B., Hoffmann, B.D., Lach, L., Lattke, J., Leponce, M., Lessard, J.P., Longino, J., Lucky, A., Majer, J., Menke, S.B., Mezger, D., Mori, A., Munyai, T. C., Paknia, O., Pearce-Duvet, J., Pfeiffer, M., Philpott, S.M., de Souza, J.L.P., Tista, M., Vasconcelos, H. L., Vonshak, M. \& Parr, C.L. (2015). Climate mediates the effects of disturbance on ant assemblage structure. Proceedings of the Royal Society of London, Series B, Biological sciences, 282: 20150418. doi: 10.1098/rspb.2015.0418

GOP, (1999). Biodiversity Action Plan of Pakistan. Prepared with support from IUCN/WWF and financed by World Bank/ GEF, $78 \mathrm{p}$

Guénard, B., Weiser, M., Gomez, K., Narula, N. \& Economo, E.P. (2017). The Global Ant Biodiversity Informatics (GABI) database: a synthesis of ant species geographic distributions. Myrmecological News, 24: 83-89.

Guérin-Méneville, F.E. (1844). Iconographie du règne animal de G. Cuvier, ou représentation d'après nature de l'une des espèces les plus remarquables, et souvent non encore figurées, de chaque genre d'animaux. Insectes. Paris: J. B. Baillière, $576 \mathrm{p}$ Janicki, J., Narula, N., Ziegler, M., Guénard, B. \& Economo, E.P. (2016). Visualizing and interacting with large-volume biodiversity data using client-server web-mapping applications: The design and implementation of antmaps.org. Ecological Informatics, 32: 185-193. doi: 10.1016/j.ecoinf.2016.02.006

Jerdon, T.C. (1851). A catalogue of the species of ants found in Southern India. Madras Journal of Literature and Science, 17: 103-127. 
Karavaiev, V. (1911). Ameisen aus Transkaspien und Turkestan. Trudy Russkago Entomologicheskago Obshchestva, 39: 1-72.

Laciny, A., Pal, A. \& Zettel, H. (2015). Taxonomic notes on the ant genus Diacamma Mayr, 1862 (Hymenoptera: Formicidae), part 1. Zeitschrift der Arbeitsgemeinschaft Österreichischer Entomologen, 67: 83-136.

Latreille, P.A. (1798). Essai sur l'histoire des fourmis de la France. Brive: F. Bourdeaux, 50 p

Latreille, P.A. (1802). Histoire naturelle des fourmis, et recueil de mémoires et d'observations sur les abeilles, les araignées, les faucheurs, et autres insectes. Paris: Impr. Crapelet (chez T. Barrois), $\mathrm{xvi}+445 \mathrm{p}$

Linnaeus, C. (1758). Systema naturae per regna tria naturae, secundum classes, ordines, genera, species, cum characteribus, differentiis, synonymis, locis. Tomus I. Editio decima, reformata. Holmiae [= Stockholm]: Laurentii Salvii, 824 p

Li-Zhong, H. (2006). List of Chinese Insects. Vol. IV. Guangzhou: Sun Yat-sen University Press, 540 p

Mani, M.S. \& Singh, S. (1962). Entomological Survey of Himalya. Journal of the Bombay Natural History Society, 59: 84-85.

Mayr, G. (1855). Formicina austriaca. Beschreibung der bisher im österreichischen Kaiserstaate aufgefundenen Ameisen, nebst Hinzufügung jener in Deutschland, in der Schweiz und in Italien vorkommenden Arten. Verhandlungen des Zoologisch-Botanischen Vereins in Wien 5: 273-478.

Mayr, G. (1861). Die europäischen Formiciden. Nach der analytischen Methode bearbeitet. Wien: C. Gerolds Sohn, 80 p

Mayr, G. (1866a). Diagnosen neuer und wenig gekannter Formiciden. Verhandlungen der Kaiserlich-Königlichen Zoologisch-Botanischen Gesellschaft in Wien, 16: 885-908.

Mayr,G.(1866b).MyrmecologischeBeiträge.Sitzungsberichte der Kaiserlichen Akademie der Wissenschaften in Wien. Mathematisch-Naturwissenschaftliche Classe. Abteilung I, 53: 484-517.

Mayr, G. (1879). Beiträge zur Ameisen-Fauna Asiens. Verhandlungen der Kaiserlich-Königlichen ZoologischBotanischen Gesellschaft in Wien, 28: 645-686.

Mayr, G. (1897). Formiciden aus Ceylon und Singapur. Természetrajzi Füzetek, 20: 420-436.

Menozzi, C. (1939). Formiche dell'Himalaya e del Karakorum raccolte dalla Spedizione italiana comandata da S. A. R. il Duca di Spoleto (1929). Atti della Società Italiana di Scienze Naturali e del Museo Civico di Storia Naturale di Milano, 78: 285-345.

Nylander, W. (1846). Adnotationes in monographiam formicarum borealium Europae. Acta Societatis Scientiarum Fennicae, 2: 875-944.
Nylander, W. (1849). Additamentum alterum adnotationum in monographiam formicarum borealium. Acta Societatis Scientiarum Fennica, 3: 25-48.

Özdikmen, H. (2010). New names for the preoccupied specific and subspecific epithets in the genus Pheidole Westwood, 1839 (Hymenoptera: Formicidae). Munis Entomology \& Zoology Journal, 5: 804-806.

Paknia, O. \& Pfeiffer, M. (2011). Steppe versus desert: multi-scale spatial patterns in diversity of ant communities in Iran. Insect Conservation and Diversity, 4: 297-306. doi: 10.1111/j.1752-4598.2011.00136.x

Pfeiffer, M., Mezger, D. \& Dyckmans, J. (2013). Trophic ecology of tropical leaf litter ants (Hymenoptera Formicidae) - a stable isotope study in four types of Bornean rain forest. Myrmecological News, 19: 31-41.

Pisarski, B. (1967). Fourmis (Hymenoptera: Formicidae) d'Afghanistan récoltées par M. Dr. K. Lindberg. Annales Zoologici (Warsaw), 24: 375-425.

Radchenko, A.G. \& Elmes, G.W.(1998). Taxonomic revision of the ritae species-group of the genus Myrmica (Hymenoptera, Formicidae). Vestnik Zoologii, 32: 3-27.

Radchenko, A. G. \& Elmes, G. W. (1999). Ten new species of Myrmica (Hymenoptera, Formicidae) from the Himalaya. Vestnik Zoologii, 33: 27-46.

Radchenko, A.G. \& Elmes, G.W. (2001). A taxonomic revision of the ant genus Myrmica Latreille, 1804 from the Himalaya (Hymenoptera, Formicidae). Entomologica Basiliensia, 23: 237-276.

Radchenko, A.G. \& Elmes, G.W. (2010). Myrmica ants of the Old World. Fauna Mundi, 3: 1-789.

Rasheed, M. T., Bodlah, I., Gull e Fareen, A. \& Huang, X. (2018). First record of Leptogenys hysterica Forel, 1900 (Hymenoptera: Formicidae: Ponerinae) from Pakistan. Journal of Threatened Taxa, 10: 13032-13036. doi: 10.11609/ jott.4036.10.15.13032-13036

Ruzsky, M. (1902). Neue Ameisen aus Russland. Zoologische Jahrbücher. Abteilung für Systematik, Geographie und Biologie der Tiere, 17: 469-484.

Santschi, F. (1927). A propos du Tetramorium caespitum L. Folia Myrmecologica et Termitologica, 1: 52-58.

Saunders, W. W. (1842). Descriptions of two hymenopterous insects from northern India. Transactions of the Entomological Society of London, 3: 57-58.

Schödl, S. (1998). Taxonomic revision of Oriental Meranoplus F. Smith, 1853 (Insecta: Hymenoptera: Formicidae: Myrmicinae). Annalen des Naturhistorischen Museums in Wien. B, Botanik, Zoologie, 100: 361-394.

Seifert, B. \& Schultz. R. (2009). A taxonomic revision of the 
Formica rufibarbis Fabricius, 1793 group (Hymenoptera: Formicidae). Myrmecologische Nachrichten, 12: 255-272.

Seifert, B. (1992). A taxonomic revision of the Palaearctic members of the ant subgenus Lasius s.str. (Hym.: Formicidae). Abhandlungen und Berichte des Naturkundemuseums Görlitz, 66: 1-67.

Seifert, B. (2003). The ant genus Cardiocondyla (Insecta: Hymenoptera: Formicidae) - a taxonomic revision of the C. elegans, C. bulgarica, C. batesii, C. nuda, C. shuckardi, C. stambuloffii, C. wroughtonii, C. emeryi, and C. minutior species groups. Annalen des Naturhistorischen Museums in Wien. B, Botanik, Zoologie, 104: 203-338.

Seifert, B. (2008). Cardiocondyla atalanta Forel, 1915, a cryptic sister species of Cardiocondyla nuda (Mayr, 1866) (Hymenoptera: Formicidae). Myrmecological News, 11: 43-48.

Seifert, B., Okita, I. \& Heinze, J. (2017). A taxonomic revision of the Cardiocondyla nuda group (Hymenoptera: Formicidae). Zootaxa, 4290: 324-356. doi: 10.11646/zootaxa.4290.2.4

Shuckard, W.E. (1840). Monograph of the Dorylidae, a family of the Hymenoptera Heterogyna. (Concluded from $p$. 271.) Journal of Natural History, 5: 315-328.

Smith, F. (1858). Catalogue of hymenopterous insects in the collection of the British Museum. Part VI. Formicidae. London: British Museum, $216 \mathrm{p}$

Smith, F. (1878). Scientific results of the Second Yarkand Mission; based upon the collections and notes of the late Ferdinand Stoliczka, Ph.D. Hymenoptera. Calcutta: Superintendent of Government Printing (Government of India), $22 \mathrm{p}$

Umair, M., Zia, A., Naeem, M. \& Chaudhry, M. T. (2012). Species composition of ants (Hymenoptera: Formicidae) in Potohar Plateau of Punjab province, Pakistan. Pakistan Journal of Zoology, 44: 699-705.

Walker, F. (1859). Characters of some apparently undescribed Ceylon insects. [part]. Annals and Magazine of Natural History, 3: 370-376.
Ward, P.S. (2001). Taxonomy, phylogeny and biogeography of the ant genus Tetraponera (Hymenoptera: Formicidae) in the Oriental and Australian regions. Invertebrate Systematics, 15: 589-665.

Weber, N.A. (1947). A revision of the North American ants of the genus Myrmica Latreille with a synopsis of the Palearctic species. Annals of the Entomological Society of America, 40: 437-474.

Westwood, J.O. (1839). An introduction to the modern classification of insects; founded on the natural habits and corresponding organisation of the different families. Volume 2. Part XI. London: Longman, Orme, Brown, Green and Longmans, 193-224.

Wetterer J.K. (2009). Worldwide spread of the ghost ant, Tapinoma melanocephalum (Hymenoptera: Formicidae). Myrmecological News, 12: 23-33.

Wetterer, J.K. (2012). Worldwide spread of the moorish sneaking ant, Cardiocondyla mauritanica (Hymenoptera: Formicidae). Sociobiology, 59: 985-997.

Wilson, E.O. (1955). A monographic revision of the ant genus Lasius. Bulletin of the Museum of Comparative Zoology, 113: 1-201.

Yahya, B.E., Yamane, S. \& Mohamed, M. (2009). Morphological and behavioral characters of the two species groups of the ant genus Myrmicaria (Insecta: Hymenoptera: Formicidae: Myrmicinae) from Southeast Asia. Species Diversity, 14: 249-265.

Yamane, S. (2009). Odontoponera denticulata (F. Smith) (Formicidae: Ponerinae), a distinct species inhabiting disturbed areas. Ari, 32: 1-8.

Yasumatsu, K. \& Brown W.L.Jr, (1957). A second look at the ants of the Camponotus herculeanus group in eastern Asia. Journal of the Faculty of Agriculture, Kyushu University, 11: 45-51.

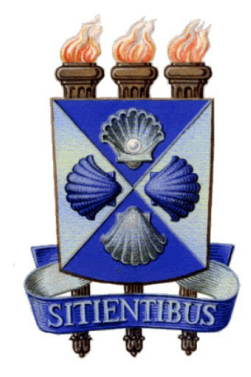

